\title{
Stage III Gastric Cancer AJCC v7
}

National Cancer Institute

\section{Source}

National Cancer Institute. Stage III Gastric Cancer A/CC v7. NCI Thesaurus. Code C89860.

Stage III includes: IIIA (T4a, N1, M0); (T3, N2, M0); (T2, N3, M0); IIIB (T4b, N0, M0); (T4b, N1, M0); (T4a, N2, M0); (T3, N3, M0); IIIC (T 4b, N2, M0); (T 4b, N3, M0); (T4a, N3, M0). T2: Tumor invades the muscularis propria. T3: Tumor penetrates subserosal connective tissue without invasion of visceral peritoneum or adjacent structures. T4a: Tumor invades serosa (visceral peritoneum). T4b: Tumor invades adjacent structures. N0: No regional lymph node metastasis. N1: Metastasis in 1-2 regional lymph nodes. N2: Metastasis in 3-6 regional lymph nodes. N3: Metastasis in seven or more regional lymph nodes. M0: No distant metastasis. (AJCC 7th ed.) 